\title{
Probabilistic modelling of ambient noise in single-cell omics data
}

\section{Authors:}

Caibin Sheng ${ }^{11}$, Rui Lopes ${ }^{1 *}$, Gang $\mathrm{Li}^{1^{*}}$, Sven Schuierer ${ }^{2}$, Annick Waldt ${ }^{2}$, Rachel Cuttat ${ }^{2}$, Slavica Dimitrieva ${ }^{1}$, Audrey Kauffmann ${ }^{1}$, Eric Durand ${ }^{1}$, Giorgio G. Galli', Guglielmo Roma ${ }^{2}$, Antoine de Weck ${ }^{1 \#}$

\section{Affiliations:}

${ }^{1}$ Disease area Oncology, Novartis Institute for Biomedical Research, CH-4002 Basel, Switzerland.

${ }^{2}$ Chemical Biology and Therapeutics, Novartis Institute for Biomedical Research, CH-4002 Basel, Switzerland.

"These authors contributed equally: Rui Lopes, Gang Li.

\#Corresponding authors. Email: caibin.sheng@novartis.com (C.S.); antoine.deweck@novartis.com (A.d.W.).

\section{Abstract}

Droplet-based single-cell omics, including single-cell RNA sequencing (scRNAseq), single-cell CRISPR perturbations (e.g., CROP-seq), and single-cell protein and transcriptomic profiling (CITE-seq) hold great promise for comprehensive cell profiling and genetic screening at the single-cell resolution. However, these technologies suffer from substantial noise, among which ambient signals present in the cell suspension may be the predominant source. Current models to address this issue are generally data-driven, highly technology-specific, and relatively scRNAseqcentric. while a universal model to describe the noise across these technologies may reveal this common source, improving the denoising accuracy. To this end, we explicitly examined these unexpected signals in multiple datasets across dropletbased technologies, summarised a predictable pattern, and developed single-cell Ambient Remover (scAR) - a hypothesis-driven machine learning method to predict and remove ambient signals at the molecular level. We demonstrated scAR's performance on three technologies - single-cell CRISPR screens, CITE-seq, and scRNAseq along with the state-of-the-art single-technology-specific approaches. scAR showed high denoising accuracy for each type of dataset. 


\section{Introduction}

Single-cell RNA sequencing (scRNAseq) enables researchers to investigate transcriptomes at single-cell resolution, improving our understanding of cellular heterogeneity and interactions between single cells and the microenvironment. Recent efforts have extended scRNAseq beyond transcriptomes by encoding additional layers of information, resulting in versatile tools for single-cell omics. For instance, by combining functional screens with scRNAseq, single-cell CRISPR screens have enabled the interrogation of multiple biological nodes in a single experiment ${ }^{1-3}$. By combining ssDNA-barcoded-antibodies with scRNAseq, CITE-seq has simultaneously quantified mRNA and surface proteins for immunophenotyping ${ }^{4}$. The most recent effort has combined both technologies to enable multimodal profiling of transcriptome and surface proteins in response to gene perturbations in cancer cells ${ }^{5}$.

Applying these pioneering technologies is challenging despite the exciting concepts and anticipated potential. One outstanding reason is the vast presence of measurement noise. Various technical factors, such as ambient contamination ${ }^{6,7}$, amplification bias $^{8}$, and index swapping ${ }^{9}$ generate noise in single-cell omics experiments. Many methods have been proposed to deal with the background signals and have facilitated the application of single-cell technologies ${ }^{6,7,10-12}$. Most of them are specific to transcriptome data, ${ }^{6,70,11}$, and several specialise in cleaning protein data in CITE-seq ${ }^{12,13}$, while few focus on recovering identity barcodes from noisy observations in single-cell feature-barcoded technologies (such as single-cell CRISPR screens and cell indexing ${ }^{14-16}$ ). These approaches are generally not transferrable between technologies. However, conceptually, the mentioned singlecell technologies have little technical difference in capturing the respective molecules (i.e., mRNA, sgRNA, expressed barcodes, and antibodies). All relevant experimental objects (cells and molecules) undergo similar processes, such as droplet formation, cell lysis, library construction, and sequencing. Background noise likely originates in a similar (if not identical) way in each of these technologies, meaning an ideal model can, in principle, describe the common sources of the artefacts in a non-technologyspecific manner. Unfortunately, no such algorithm has been proposed so far.

Here, we took a bottom-up strategy - investigating ambient signals across technologies, summarising a predictable pattern, and building a statistical deep 
learning model, termed single cell ambient remover (scAR) to model the common noise sources. We first evaluated the potential noise source across technologies. Cell-free transcripts have been observed in empty droplets ${ }^{17}$. They may arise from ambient mRNAs in single-cell suspension, which likely originates from damaged cells (e.g., caused by cell lysis) ${ }^{6,18,19}$. This hypothesis suggests that ambient mRNAs may not be completely random but deterministic signals to a certain extent. Indeed, the frequencies of cell-free transcripts correlate well with their cellular counterparts ${ }^{6}$. These motivated us to systematically evaluate the ambient signal hypothesis in multiple single-cell technologies, which revealed a predictable pattern of ambient noise. Exiting single-cell analysis methods which implement probabilistic machine learning technologies, including totalVI ${ }^{12}, \mathrm{DCA}^{10}, \operatorname{scVI}^{11}, \operatorname{Decont}^{7}$, and $\operatorname{scVAE}^{20}$, have shown significant advantages in modelling single-cell omics data. Together with the ambient signal hypothesis, these inspired us to build a universal probabilistic model to describe this type of noise across technologies. To highlight the generality of our approach, we applied scAR to multiple kinds of single-cell datasets - including an internal single-cell CRISPR screens dataset and several public CITE-seq datasets and scRNAseq datasets - and demonstrated scAR's accuracy in comparison with competing technology-specific methods where available. 


\section{Results}

\section{The scAR model.}

ScAR uses a latent variable model to represent the biological and technical components in the observed count data (Fig. 1). It is designed under the ambient signal hypothesis, which assumes that ambient signals originate from broken cells during sample preparation, are homogenised in the single-cell solution (ambient signal pool) and contaminate cell-containing and cell-free droplets (Fig. 1a). Mathematically speaking, we assume that ambient signals in cell-containing and cellfree droplets are drawn from a distribution (Binomial, or Poisson, or zero-inflated Poisson) with fixed probabilities per feature (denoted as ambient frequencies, $\alpha$ ). These probabilities can be estimated by averaging cell-free droplets as their counts all are ambient signals. For cell-containing droplets, we introduce two hidden variables, noise ratios $(\varepsilon)$ and native expression frequencies $(\beta)$, to represent the total contamination level per cell and normalised 'true' expression per cell, respectively (Fig. 1b). ScAR simultaneously infers $\varepsilon$ and $\beta$ using the variational autoencoder (VAE) framework ${ }^{21-23}$ (Fig. 1b, Methods).

We use the optimised variables $\varepsilon$ and $\beta$ as well as the sequencing depth to estimate the 'theoretical' gene expression, which is denoted as denoised counts and can be directly used by other well-established tools for downstream analysis. In addition, in several feature barcode technologies, such as CROP-seq and CellTagging ${ }^{14}$, the presence/absence of native signals is more critical information than the actual level. To reflect this, scAR also outputs a matrix of Bayes factors representing the likelihood of whether 'true' native signals are present in raw counts. 

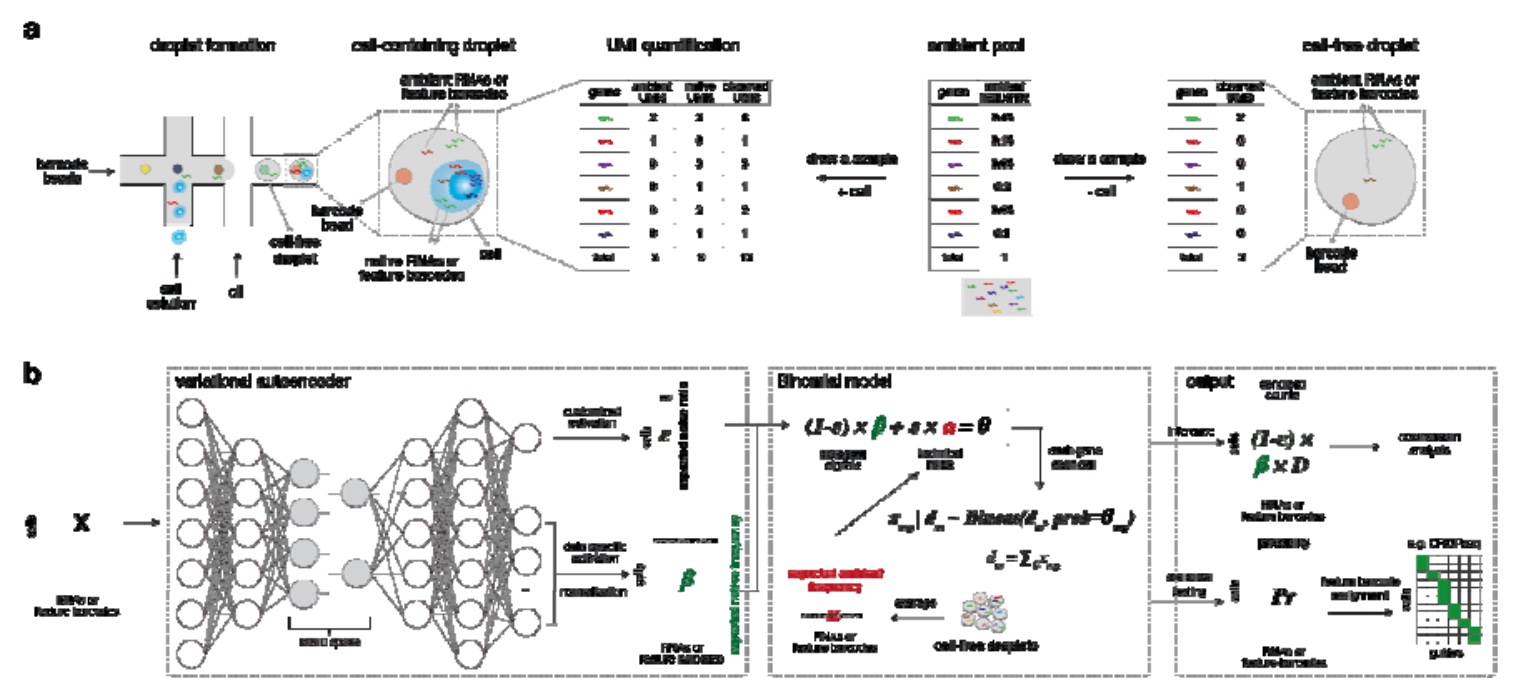

Fig. 1 | The overview of scAR. scAR is a hypothesis-driven denoising model for droplet-based single-cell omics technologies. a, Demonstration of the ambient signal hypothesis. During the preparation of the single-cell solution, RNA or protein counts are released upon cell lysis and consequently encapsulated by droplets with or without a cell. In the case of a cell, these exogenous molecules are mixed with native ones and barcoded by the same 10x bead, resulting in ravelling count data. Under this assumption, the ambient signals in both cell-containing and cell-free droplets are drawn from the same pool. UMI stands for the unique molecular identifier. For illustration, the reddish-purple, the light green, and the mixture indicate ambient signals, native signals, and observed counts, respectively. b, scAR takes raw count matrices of RNA or protein as input and learns two sets of parameters $(\varepsilon$ and $\beta$ ) through the variational autoencoder. $\varepsilon$, a column vector represents noise ratios per cell and $\beta$, a matrix represents cell-wise native frequencies of RNAs or proteins. $\alpha$, a row vector represents the ambient frequencies of RNAs or proteins, which is empirically estimated by averaging cell-free droplets. scAR uses a unique a for all cells in a single experiment. The observed raw counts are modelled using a Binomial model containing known parameters $\alpha$ and sequencing depth $\mathrm{D}$ and two hidden variables $\varepsilon$ and $\beta$. We optimised $\varepsilon$ and $\beta$ by minimising the reconstruction errors and K-L divergence (Methods). ScAR outputs two matrices, a denoised count matrix and a matrix of Bayes factors. The latter represents the likelihood that native signals are present in given observed counts. The meaning of colour codes is the same as a.

\section{Evaluation of ambient signal hypothesis.}

We conducted a case study that combined CROP-seq and bulk sequencing to evaluate the ambient signal hypothesis (Fig. 2a and Methods). We designed a viral pool of 99 sgRNAs targeting 13 different genes (supplementary table I), most of them being essential in MCF7 cells ${ }^{24,25}$ (supplementary Fig. 1a). We infected MCF7 cells expressing dCas9-KRAB with the lentiviral libraries at 0.3 multiplicity of infection to ensure a majority ( $84 \%$ after selection) of single infections. Excessive sgRNAs in a cell are supposed to be ambient signals. Cells were harvested at various time points post-transduction and split into two portions, with one portion taken for 10x scRNAseq and the other for bulk sequencing of sgRNAs, which revealed the 
frequencies of sgRNA libraries in the samples. In addition, the downregulation of target genes by CRISPRi provided an additional way to assess the identification of truly integrated sgRNAs.

We first examined the raw sgRNA counts in cells and observed the strong presence of ambient counts (Fig. 2b). 25 distinct sgRNAs were detected per cell on average, while $<=1$ sgRNA was expected because of the low multiplicity of infection. To validate whether ambient sgRNAs are correlated with their native counterparts, we compared sgRNA frequencies in bulk sequencing and cell-free droplets from CROPseq. Results showed a high correlation of sgRNA frequencies at both time points (Fig. 2c and supplementary Fig. 1b). Randomly sampled subsets of cell-free droplets (from 20 to 10000) show a high correlation (Fig. 2d). Together, these observations confirm that ambient signals are not random noise but endogenous expressioncorrelated artefacts. We next asked whether this correlation persists in other dropletbased technologies. To answer it, we checked the mRNA counts of the in-house CROP-seq experiment, protein counts of a public CITE-seq ${ }^{26}$, and sgRNA counts of another three single-cell CRISPR screens ${ }^{3,26,27}$. Surprisingly, feature frequencies (i.e., mRNA, sgRNA, or protein) in as few as $\sim 50$ cell-free droplets consistently show a high correlation to that of the cell-containing droplets despite the varying numbers (from 32 20,000) of features (Fig. 2e-f and supplementary Fig. 2). Altogether, these indicate that the ambient signals are systematic noise across single-cell omics technologies, and they are strongly correlated with native signals in the pooled cells. Thus, building scAR on the ambient hypothesis is rational. 


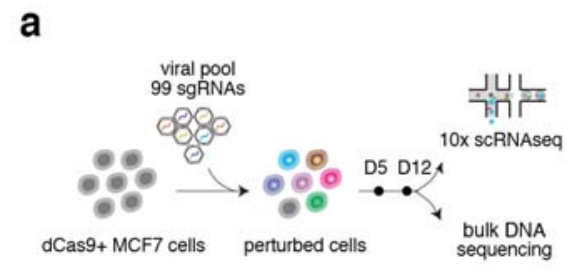

b
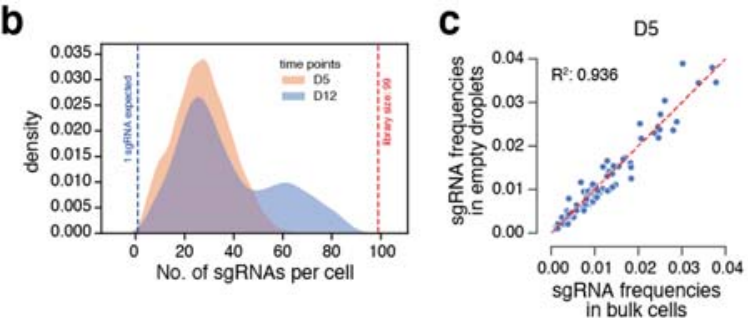

d

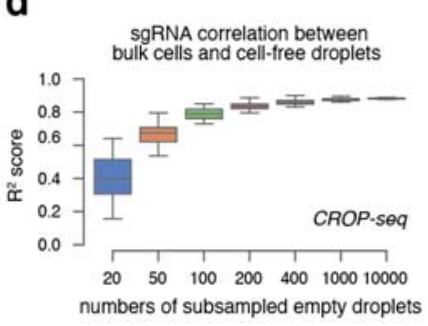

e

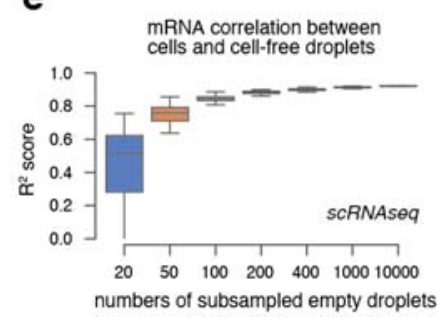

$\mathbf{f}$

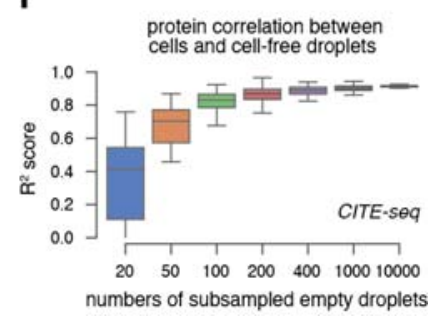

Fig. 2 | Examination of the ambient signal hypothesis. We systematically evaluated the ambient signal hypothesis in various types of single-cell omics data. a, Demonstration of the experimental design (Methods). $\mathbf{b}$, Distribution of distinct sgRNAs per cell in the raw counts. The X-axis represents the number (not UMl count) of distinct sgRNAs per cell; the $y$-axis shows the density. The size of lentivirus libraries and the expected number of sgRNA are highlighted by dashed lines. Time points were indicated. c, Scatterplot of sgRNA frequencies between two independent experiments. The $X-$ axis represents sgRNA frequencies in bulk DNA sequencing; Y-axis represents sgRNA frequencies obtained by averaging RNA counts in cell-free droplets from 10x scRNAseq. Each dot represents an sgRNA, the red dashed lines represent $y=x$, and coefficients of determination ( $R^{2}$ scores) is shown. $\mathbf{d}$, Coefficients of determination ( $R^{2}$ scores) show the correlation of sgRNA frequencies between bulk cells and randomly sampled cell-free droplets. The X-axis shows the numbers of randomly sampled cell-free droplets. Forty samplings were performed in each group. e-f, Similar to Fig. 2d, correlation analysis of mRNA counts and protein counts (f) between cell-containing and -free droplets.

\section{scAR enables accurate guide calling in single-cell CRISPR screens.}

Single-cell CRISPR screens (including Perturb-seq ${ }^{28}, \mathrm{CRISP}_{-} \mathrm{seq}^{29}$, and CROPseq $^{1,2}$ ) are powerful tools for functional screening with single-cell transcriptome readout, yet, few methods focused on dealing with the noise, although accurate guide calling is a key to data quality and result interpretation. Most of the current studies considered the most highly expressed guides as the true signal and relatively low counts as background noise ${ }^{1,2,28-30}$, which we found inappropriate, especially when a few guides were predominantly enriched in the libraries (data not shown), as these guides could cause a significant amount of ambient signal and mask the true native signals.

We, therefore, asked whether scAR could distinguish the ambient signals and improve the guide calling. To test it, we applied scAR to the CROP-seq dataset and benchmarked it against the baseline approach, termed as naïve assignment. Here, we did not involve any subjective filters to either naïve assignment or scAR-based 
assignment for the benchmarking purpose. All cells that passed the default gene and cell filtering in Cellranger were included for downstream analysis (Methods). By naïve assignment, $~ 80 \%$ cells (20076 out of 25248, D5 and D12 combined) were assigned to unique guides, and $20 \%$ (5170 out of 25248, D5 and D12 combined) cells were assigned to multiple $(>=2)$ guides due to equal expression (Fig. 3a). 'Multiple-infected' cells were generally filtered out before downstream analysis in CROP-seq experiments; in other words, naïve assignment caused loss of $\sim 20 \%$ cells. scAR estimated the expected ambient counts then compared them to the observed counts via hypothesis testing to evaluate the probability of the presence of native signals. It nearly assigned all cells to a single guide (Fig. 3a) despite $\sim 20 \%$ cells with equally expressed guides. We next examined the cell fraction partitioned by distinct sgRNAs after guide assignment. This fraction was expected to be identical to sgRNA frequencies in bulk sequencing since both reflected sgRNA libraries in the cell pool. scAR-resulting cell fractions were highly correlated with sgRNA frequencies in bulk sequencing $\left(R^{2}=0.879\right.$ at $\mathrm{D} 5$ and $R^{2}=0.910$ at $\left.\mathrm{D} 12\right)$, while naïve assignment preferably over-assigned a few sgRNAs of highest expression $\left(R^{2}=0.369\right.$ at D5 and $R^{2}=0.861$ at D12, Fig. 3b-c).

Pooled screens with proliferation readouts led to the discovery of many potential drug targets in Oncology $24,25,31$, where the gene dependencies were generally calculated on millions of reads per target ${ }^{32}$. We wondered whether a few hundred cells per guide in the CROP-seq experiment could capture the gene dependencies given the high accuracy of guide assignment by scAR. To check it, we first used bulk sequencing data of multiple time points (D0, D5, D7, D9, and D12) to fit an exponential model for each guide (see our previous report ${ }^{3}$ ). The growth rate in the model reflected the fitness score of each guide, which were consistent with wellestablished datasets (supplementary Fig. 1a). We then took the same procedure but with the cell fraction data (CROP-seq experiment) of three time points (D0, D5, and D12) and compared the resulting dependency scores (Fig. 3d). Excitingly, most of the guides show comparable fitness scores between these two datasets, despite the small sample sizes ( 92 cells per guide) and limited time points. This confirms the high accuracy of scAR-based assignments.

Next, we checked the expression levels of targeted genes in cells with certain guides assigned exclusively by either naïve approach or scAR (Fig. $3 e-g$ ). We considered the cells assigned by both naïve and ScAR as the positive control and cells assigned 
with CTL sgRNAs by both naïve and scAR as the negative control. In an example shown in Fig. 3e, naïve assignment assigned 263 cells to TP53_g3, 245 cells among which were mutually assigned to the same guide by scAR. These 245 cells show downregulation of TP53, suggesting the effectiveness of this guide. However, the remaining naïve assigned 18 cells show similar expression as in CTL cells, suggesting that these cells may not integrate TP53_g3. More importantly, another 58 TP53_g3 cells, exclusively identified by scAR, show low expression as in the mutually assigned cells. Similarly, for the other example, FOXA1_g4 (Fig. 3f), 15 cells identified by scAR but missed by naïve assignment show a similar expression pattern as the mutually assigned cells. To systematically assess and visualise the difference, we performed a t-test on the expression of targets among these subgroups for each guide. We visualized both t-statics and p-values using dotplots (Fig. $3 g$ and supplementary Fig. 3a). In total, scAR rescued $\sim 20$ sgRNA groups at each time point which were missed by naïve assignment as confirmed by statical confidences. In comparison, fewer than two sgRNA groups were missed by ScAR at each time point, compared to naïve assignment. In addition, we counted the cell numbers of each subgroup and visualised the difference (supplementary Fig. 3b). Naïve assignment over-assigned cells to a few guides, such as CCND1_g3, CTL_g5, and YAP1_g1, likely due to their more substantial ambient presence than other guides (supplementary Fig. 3c), whereas the power of scAR to identify ambient sgRNAs by their distribution ensured unbiased assignment. It recovered more than ten cells per group for most of the target groups (supplementary Fig. 3b). Together, by inspecting the guide assignment in the CROP-seq dataset, we showed that ScAR significantly improved the assignment accuracy in feature barcode technologies, where the presence rather than the quantity of native signals was the key information. 
a
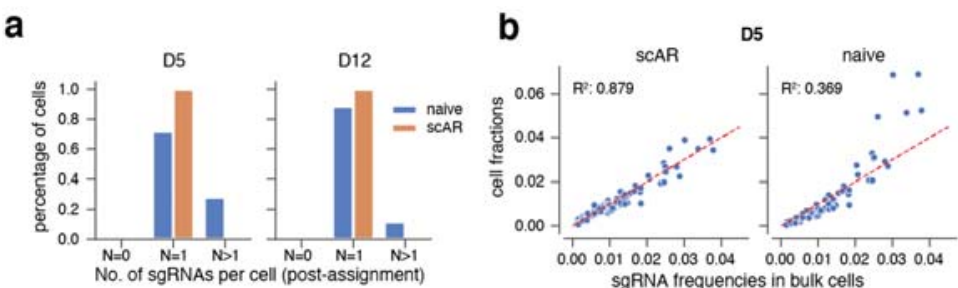

sgRNA frequencies in bulk cells

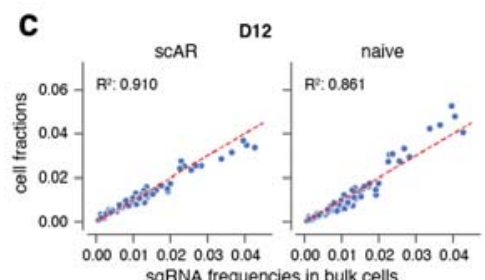

sgRNA frequencies in bulk cells

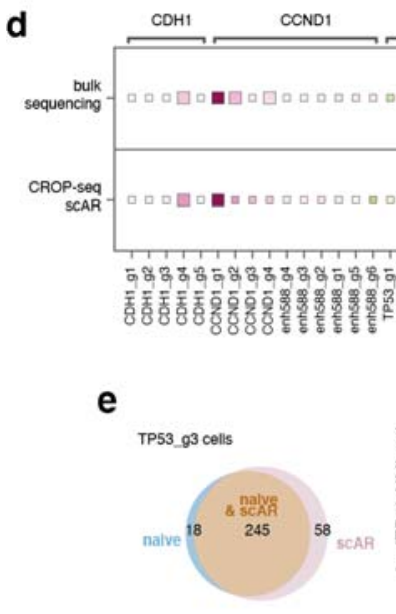

e

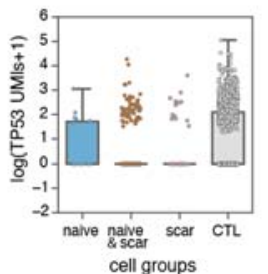

g

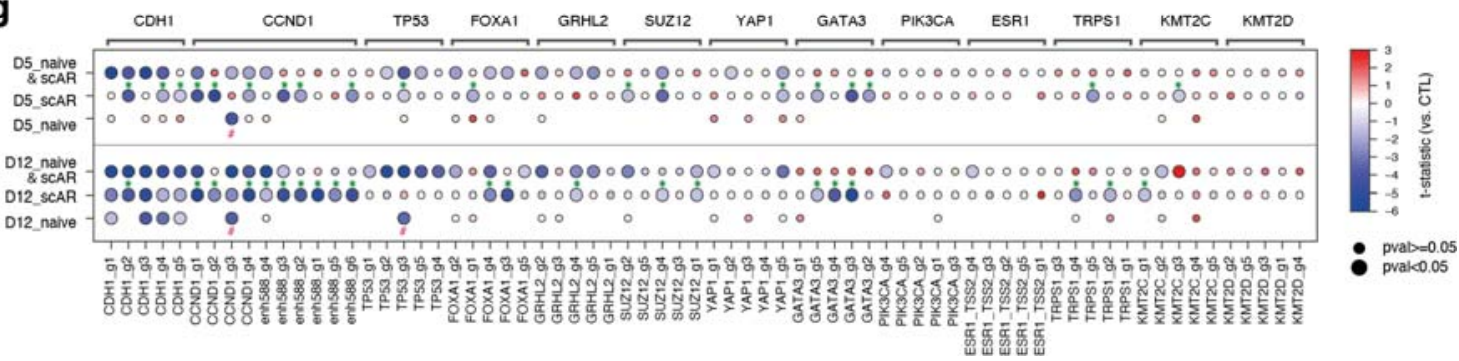

Fig. 3 | scAR enables accurate guide calling in single-cell CRISPR screens. We demonstrate that scAR-guided assignment shows superior performance compared to the widely used naive assignment. a, Distribution of null, single and multiple assignments. Cells were grouped by the number of assigned sgRNA(s). b-c, Scatterplots show the correlation of sgRNA frequencies between cells from 10x scRNAseq (post-assignment) and bulk cells at D5 (b) and D12 (c). Y-axis represents cell fractions grouped by distinct sgRNAs. The red dashed lines represent $y=x$. d, CROP-seq with scAR-guided assignment shows comparable results of gene dependency analysis to bulk screening. Green and maroon indicate cancer cell promotion and cancer growth inhibition, respectively. Sizes of squares indicate $p$ values. e-f, Two selected guides demonstrate scAR's sensitivity and specificity. The Venn diagrams show the number of cells assigned with TP53_g3 (e) at D5 and FOXA1_g4 (f) at D12. Exclusive naïve assignment, exclusive scAR assignment, and accordant assignment are marked with blue, purple, and brown, respectively. The boxplots show the expression of TP53 or FOXA1 in these subgroups. Y-axis represents the log-transformed UMI counts after library size normalisation (Methods). Dots represent cells. Co-assigned CTL cells are used as the negative control. g, The dotplot shows the on-target downregulation. The X-axis represents guide groups. Y-axis represents subgroups of cells, separated by two time points and assignment approaches. Target genes are shown on the top. Their expression in each group is compared with that in the CTL group (centred at zero), and resulting t-statistics are guided by the dot colours (Methods, see t-statics of z-normalized expression in supplementary Fig. 4a). Blue and red indicate down- and up-regulation, respectively. The bimodal sizes of circles represent the p-values from the t-test. The asterisks $\left(^{*}\right)$ and hashes (\#) 
highlight the guide groups where scAR outperforms and underperforms naïve assignment, respectively.

\section{scAR removes the ambient protein counts in CITE-seq.}

Next, we evaluated ScAR on CITE-seq along with current protein denoising methods, such as totalVI ${ }^{12}$ and $\mathrm{DSB}^{13}$. We used a public CITE-seq dataset ${ }^{26}$ of peripheral blood mononuclear cells (PBMC5k), which were profiled with a panel of 32 antibodyconjugated oligos consisting of surface markers of B cells, T cells, Natural killer cells (NK), monocytes, and Dendritic cells (DC) (supplementary Fig. 4a). We first annotated cell types by clustering cells with transcriptome signatures (Fig. 4a and Methods) and compared protein counts per cell type before and after denoising.

As expected, ambient protein signals were observed in raw counts in every cell. Both scAR and totalVI could preserve cell-specific true signals, and scAR nearly eliminated all ambient counts, while totalVI generally missed some ambient counts in several cell types (especially monocytes and memory-like NK cells, Fig. 4b-e and supplementary Fig. 4). DSB-denoised values reflected the number of standard deviations above background noise rather than the molecular-level counts; it rescaled the true signals and hardly eliminated all ambient signals in cells given the high cell-to-cell variations (even for control antibodies, a half population was supposed to have positive values with DSB normalisation). For example, scAR cleaned most of the non-specific CD20 and CD19 in non-B cells, and totalVI also removed most of non-specific CD20 and CD19 but missed some in monocytes and memory-like NK cells. DSB rescaled both the native and ambient signals and could not clean all ambient signals in other cells (Fig. 4b, 4d, and supplementary Fig. 4b). For simplicity and fairness, we benchmark ScAR with only totalVI when comparing the counts of proteins in the following text as both are molecular level denoising approaches. Similarly, we found that ScAR nearly recognised all non-specific CD3 and PD-1 in non-T cells (Fig. 4c, 4e, and supplementary Fig. 4c), non-specific CD56 and CD335 in non-NK cells (supplementary Fig. 4d-e) as well as non-specific CD14 in non-monocytes (supplementary Fig. 4f). totalVI also showed promising performance, identifying all non-specific CD14 in non-monocytes (supplementary Fig. 4f), almost all non-specific CD56 and CD335 in non-NK cells (supplementary Fig. 4de) and most non-specific CD3 and PD-1 in non-T cells (Fig. 4c, 4e, and supplementary Fig. 4c). We also found that scAR successfully identified the lgG1 signal as noise in all cells while totalVI had a visible level of negative-positive rate 
(supplementary Fig. 4g). It is worth underlining that the expressions of ambient proteins were highly diversified (mean $9.6 \pm$ STD 43.4 per marker per cell). In some cases, the true signals were as low as background noise. For example, the naïve $T$ cells showed a similar PD-1 level as B cells, NK cells, and monocytes; while scAR retained the PD-1 signal in naïve T cells but eliminated all signals in B cells, NK cells, monocytes, and DC cells (supplementary Fig. 5a-b). To assess how much scAR corrected the ambient noise, we calculated the per cell fractions of estimated ambient counts and compared it with totalVI (supplementary Fig. 6). In addition to removing ambient counts, totalVI added extra counts, particularly to the highly expressed proteins due to its imputation functionality ${ }^{12}$, resulting in unchanged total counts and high denoising variance (noise ratio at $-0.2 \% \pm$ STD $29 \%$ ). In contrast, scAR removed more raw protein counts on average but was highly consistent in each cell type (mean $7.5 \% \pm$ STD $2.4 \%$ ).

We next compared the averaged protein levels by cell type before and after denoising and aligned them with RNA expressions (Fig. 4f). Almost all antibodies showed intense background noise at the populational level. DSB-resulted normalisation down-scaled but did not eliminate ambient signals, and totalVI preserved true signals and reduced most ambient contamination. scAR also held true signals and nearly removed all the background noise, resulting in highly specific expressions of markers. For example, scAR-denoised CD8 were exclusively present in CD8+ T cells, while after DSB- or totalVI-denoising, there was still residual CD8 in other cell types (Fig. $4 \mathrm{f}$ and supplementary Fig. 7a). Compared to DSB and totalVI, scAR-denoised CD197 and CD45RA could separate Naïve and memory T cells well (Fig. $4 \mathrm{f}$ and supplementary Fig. 7b). Overall, after scAR-denoising, protein expressions correlated stronger with corresponding RNAs, and the Spearman correlation coefficient between them was increased to $\sim 0.6$ (Fig. 4g). Together, these results demonstrate that $\mathrm{SCAR}$ is an accurate tool for protein denoising in CITE-seq. 
a

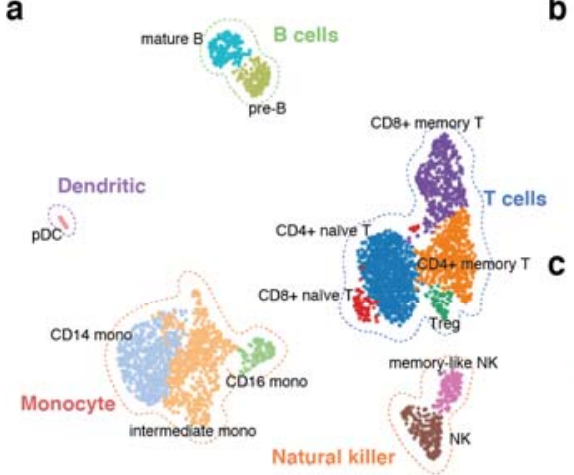

d

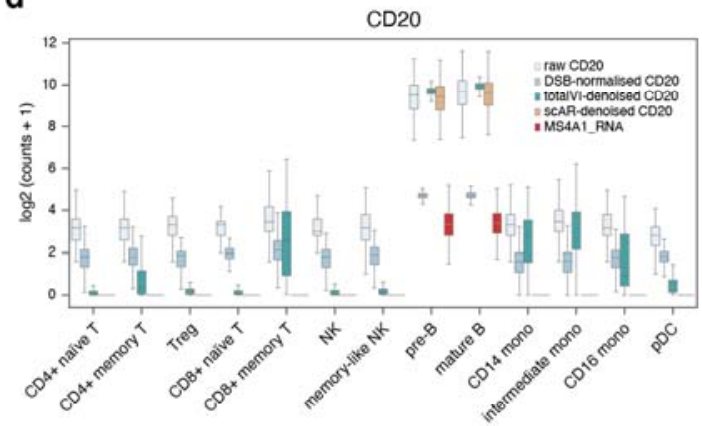

raw CD20 DSE-normalsed CD20 totalli-denoised CD20 scAR-denoised CD20
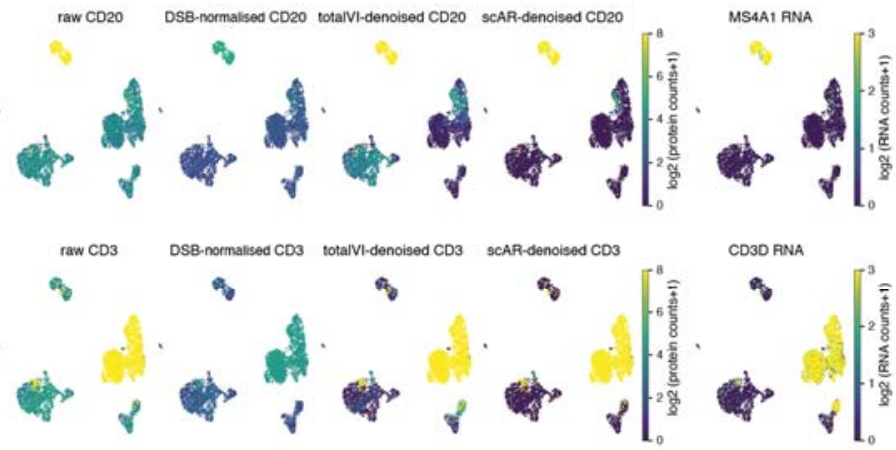

$\mathbf{f}$

f raw protein $\quad$ DSB-nomaliog

e
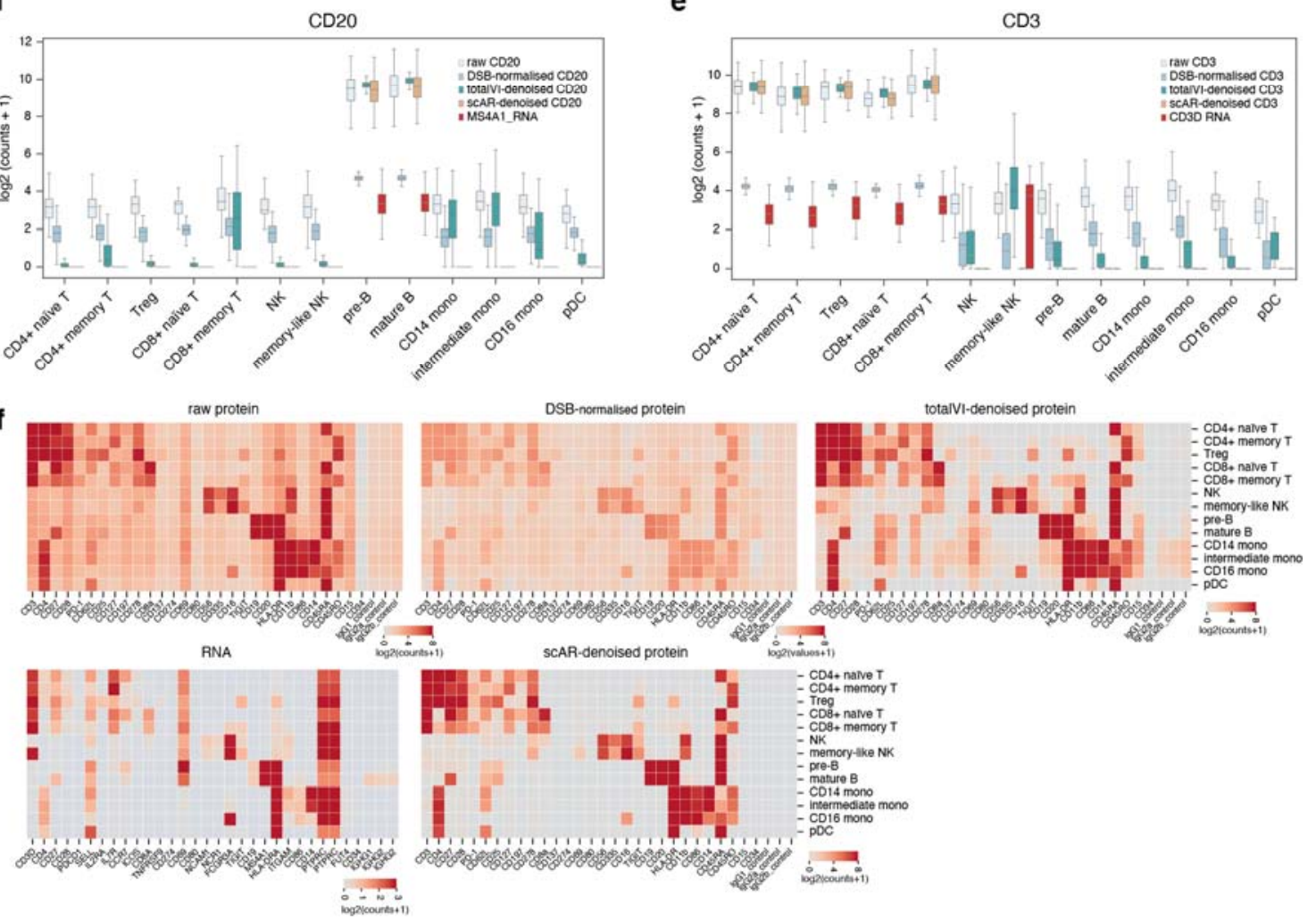

g
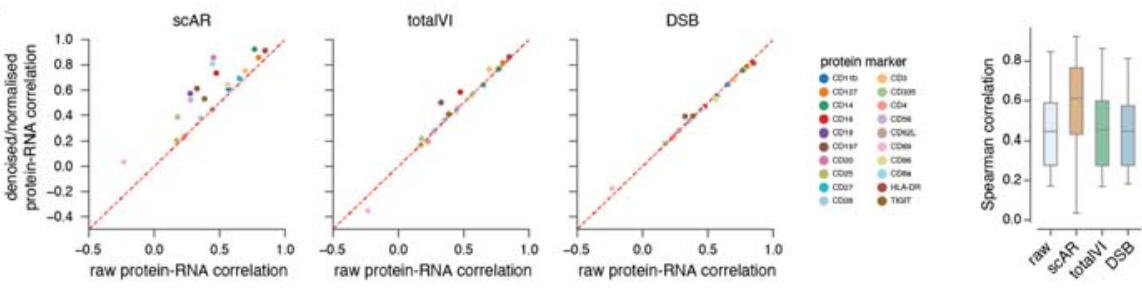

Fig. 4 | scAR removes the ambient protein counts in CITE-seq data. scAR outperforms competing protein-denoising approaches. a, UMAP of the PBMC5k dataset. Cell types are annotated using transcriptome data (Methods). b-e, Two selected examples, CD20 antibody (b, d) and PD-1 antibody (c, e), demonstrate scAR's performance. UMAPs visualise raw protein counts, DSB-normalised, totalVI-denoised, and scAR-denoised protein counts, and the corresponding RNA counts, respectively. Colour bars represent log2 (counts +1 ) (log2(normalised values +1 ) in the case of DSB), and UMAPs of protein counts use the same scaling. Boxplots show the single-cell counts grouped by cell types. $\mathbf{f}$, Heatmaps show the cell types' average protein (raw, DSB-normalised, totalVI-denoised, and scARdenoised) and RNA counts. Columns and rows represent the antibodies (or corresponding RNAs) and 
cell types. g, Scatterplots show the Spearman correlation coefficients between RNA-protein pairs before ( $x$-axis) and after ( $y$-axis) protein denoising. The red dashed line represents $y=x$. Dots represent antibodies. The rightest boxplot summarises the coefficients.

\section{scAR accurately removes ambient mRNA in scRNAseq.}

Many methods have been developed for denoising or normalising scRNAseq data $^{6,7,10,11,20,33-36}$. To demonstrate the advances of scAR, we benchmarked it with the most state-of-the-art approaches, including $\mathrm{DCA}^{10}$ and $\mathrm{scVI}{ }^{11}$. We selected a public dataset ${ }^{26}$ that sequenced pooled human HEK293T and mouse NIH3T3. In this dataset, transcripts from the other organism were unambiguously ambient contamination, providing a 'ground truth' for the evaluation. By unsupervised transcriptome clustering, we identified 7590 HEK293T cells, 8006 NIH3T3 cells and 697 mixed droplets, which contained both HEK293 and NIH3T3 cells (supplementary Fig. 8a).

Interestingly, we noticed that the compositions of ambient transcripts ( $\alpha$ in Fig. 1b) varied between different subpopulations of droplets (supplementary Fig. 8b-d and Methods). When taking different populations to calculate ambient frequencies, scAR outputted slightly different estimated contamination rates (supplementary Fig. 8e). Overall, scAR could precisely predict the cross-species ambient signals (i.e., the mouse transcripts in human cells or human transcripts in mouse cells). In comparison, the prediction for inter-species ambient signals (e.g., ambient mouse transcripts in mouse cells) depended on the inputting ambient frequencies. This suggests that a precise estimation of ambient frequencies is key to noise reduction. In addition, estimation of ambient frequencies by averaging cells yields similar results as by averaging cell-free droplets; therefore, scAR package uses the transcript frequencies in cells as a default setting to simplify the usage of scAR. However, we recommend calculating ambient frequencies using well-defined cellfree droplets to achieve the highest denoising performance.

We benchmarked these two modes of scAR along with scVI and DCA. Both human and mouse cells exhibited low exogenous contamination in the raw counts (Fig. 5a). After denoising, we observed that two modes of scAR removed all cross-species contamination (Fig. 5a-b and supplementary Fig. 9a). Nearly all mouse transcripts were identified and removed in HEK293T cells, and almost all human transcripts 
were also identified and removed in NIH3T3 cells. On the other hand, the mouse and human transcripts ratio was maintained at 1:1 in the multiplets after denoising.

In contrast, DCA and scVI did not remove the cross-species contamination (supplementary Fig. 9b-c). The ambient signal hypothesis might partially explain this. Ambient signals were not random but 'evenly' distributed (the presence of ambient transcript follows a fixed probability per transcript) in each cell. Therefore, methods, like scVI and DCA, which specialised in reducing stochastic noise, might treat the ambient signals as the baseline 'native' signals.
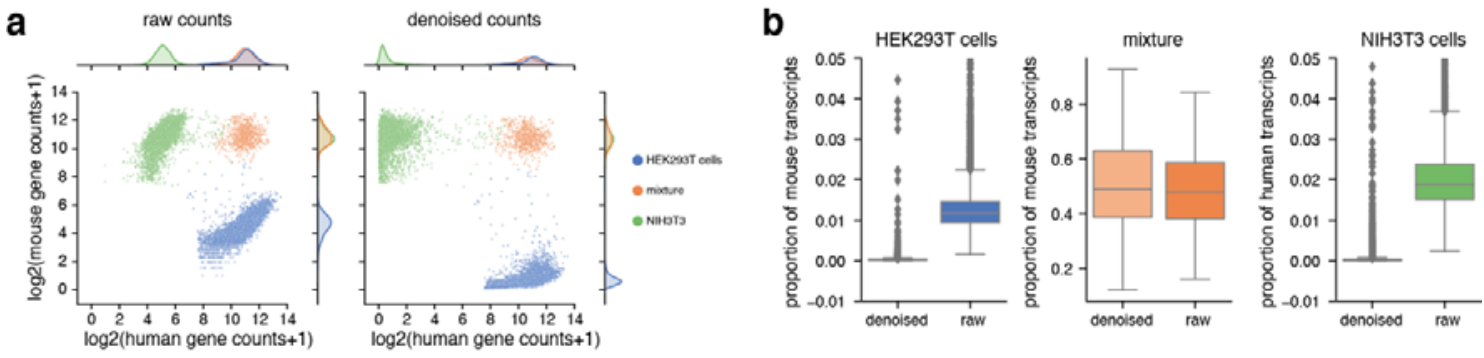

Fig. 5 | scAR eliminates ambient RNAs in scRNAseq. A public scRNAseq dataset of mixed human HEK293T and mouse NIH3T3 cells (1:1) was selected to demonstrate scAR's ability in noise reduction in transcriptome data. a, Scatterplots show transcript composition before (left) and after (right) ScAR denoising in three populations, HEK293T, NIH3T3 and multiplets. X- and y-axis show transcripts exclusively mapped to the human or mouse genome, respectively. $\mathbf{b}$, Quantification of exogenous contamination before and after denoising in three populations. Y-axis represents per cell fraction of exogenous transcripts, i.e., mouse transcript rate in all HEK293T cells and human transcript rate in all $\mathrm{NIH} 3 \mathrm{~T} 3$ cells.

\section{scAR is scalable and computationally efficient.}

Next, we benchmarked the resource efficiency by comparing the model size and runtime between scAR and similar methods. The model size (number of total parameters) is approximately linearly correlated to the number of features. Under default settings (e.g., default width and depth of neural networks), scAR has much fewer trainable parameters than totalVI, and scVI ( 0.26x and $\sim 0.54 \mathrm{x}$, respectively), meaning scAR requires less memory resource and consumes less electric power. An exception is DCA, which has $\sim 74 \%$ fewer parameters than scAR (Fig. 6a). However, training DCA is approximately $~ 55 \%$ slower than scAR under the same settings (e.g., sample size, epochs, and batch size) (Fig. 6b). It should be noted that DCA was trained on a 28-core CPU. GPU implementation of DCA can significantly shorten the training time. ScAR is slightly faster than scVI, and it saves $\sim 16 \%$ time in all test 
samples. For processing CITE-seq data, totalVI is faster than scAR as it simultaneously processes protein and mRNA data. In contrast, scAR needs to be run separately for protein and mRNA data (data not shown). Like DCA, scVI, and totalVI, scAR uses the minibatch functionality and can process a small number of cells at a time without scarifying accuracy. This makes it scalable to large-scale single-cell data even with limited GPU memory.
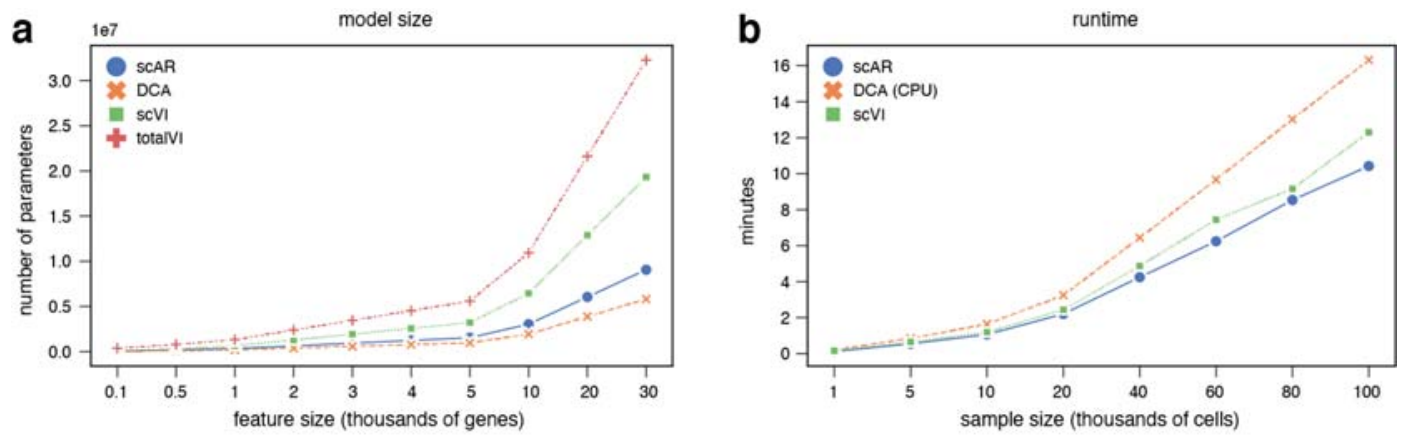

Fig. 6 | Benchmarking the efficiency of scAR. a, The lineplot shows the number of total trainable parameters under the default settings of each algorithm. $\mathbf{b}$. The lineplot shows the runtime on subsampled cells of a 1.3 million single-cell datase ${ }^{26}$. Experiments are run on the same machine and under the same parameter settings where possible. 


\section{Discussion}

Versatile single-cell omics technologies have expanded understanding of single-cell biology, and the development of new technologies is constantly pushing the boundary further. In this context, we developed ScAR to provide a reliable 'one-for-all' denoising solution for these technologies.

scAR can precisely infer the native signals for protein data in CITE-seq and mRNA data in scRNAseq. Recent approaches ${ }^{11,12,20,27,33,37}$ introduce deep learning technologies (such as $A E$ and VAE) for these tasks and show great promise. These approaches generally design noise models based on data patterns (e.g., zeroinflation) and learn the model parameters through neural networks ${ }^{11,13,33}$. In scAR, we constrain the noise model under the ambient signal hypothesis and empirically estimate a set of parameters from cell-free droplets, this roughly reduces the outputs of VAE by half (supplementary Fig. 10) and focuses the VAE on learning the other latent variables, including biology-related native expression and the noise ratios. As a result, this hypothesis-driven modelling performs better at cleaning ambient signals than those that disregard the ambient signal hypothesis (Fig. 5 and supplementary Fig. 10). Moreover, it generalises scAR to fit a broader range of droplet-based singlecell datasets, independent of the data sparsity. However, it should also be noted that other methods, particularly DCA and scVI, has stronger strength in reducing stochastic noise and cellular heterogeneity than ScAR because of their more flexible noise models (data not shown). Strictly speaking, scAR is distinct from these methods and can even be combined to achieve higher denoising performance. For example, one can consider scAR as a part of the pre-processing pipeline to remove ambient noise and subsequentially use other methods to reduce single-cell variance.

Another use case of scAR is the assignment of identity barcodes. Several single-cell omics technologies integrate exogenous unique barcodes into single-cell genome to label and/or perturb the host, such as single-cell CRISPR screens (e.g., CROP-seq, Perturb-seq and CRISP-seq $)^{1,2,28,29}$ and cell indexing ${ }^{14-16}$. This class of single-cell technologies suffer heavily from the ambient noise of identity barcodes. Unlike CITEseq and scRNAseq, the matrices of identity barcodes contain no protein or transcript signatures for cross-validation. Most of the current studies have assigned exogenous barcodes by hard filtering approaches ${ }^{28,30}$, which filter out cells with low depth and perform naïve assignment afterwards. This is not only inaccurate (Fig. 3) but also 
inefficient as it can discard as many as half of the cells despite their high quality of transcriptome readouts ${ }^{38}$. Other approaches such as MUSIC $^{38}$ and ScMAGeCK $^{39}$ proposed to link transcriptome profile to sgRNA assignment. These methods are specific to single-cell CRISPR data (unlikely suitable for the cell indexing case). On the other, there is a risk of being misled by potentially dominant transcriptional states (e.g. cell cycle). They might also fail in other cases, for example, when several nodes of the same pathway are being interrogated or when the phenotypic effect of the perturbation is low (in the case of, e.g., low effective sgRNAs or wrong time points). In contrast, scAR uses only the information of identity barcodes (i.e., sgRNAs and cell tags) and accurately evaluate the probability of ambient contamination for each barcode. This ensures a more accurate and unbiased assignment of identity barcodes. We tested scAR on single-cell CRISPR screening, but it should fit other technologies in this class as these technologies all use similar protocols to prepare, construct and sequence the barcodes (sgRNAs or identity barcodes).

Besides ambient contamination, other technical factors ${ }^{8,9}$ can also introduce background noise. We assume in ScAR that ambient source is the most predominant artefact, and in turn, this hypothesis seems to be confirmed by the outstanding performance of scAR. However, further experimental validation may still be required. In addition, in CITE-seq technology, the non-specific binding of antibodies may bring in extra noise $e^{4,13}$. This is not modelled in scAR as we consider it too specific (dependent on the antibodies and experimental cell lines) to violate the scope of scAR's generality. Moreover, identification of this noise may require dedicated welldesigned experiments (e.g., spike-in ${ }^{4}$ ), as models can hardly distinguish between specific and non-specific binding without human intervention.

Finally, we observed different contamination levels in different datasets. scAR's ability to estimate noise ratio may allow to evaluate batch effects and guide the experimental design, such as the protocols for cell fixation and washing. Furthermore, scAR has great potential in facilitating technology implementation and development in droplet-based single-cell omics. For example, single-cell analysis of accessible chromatin (scATAC-seq) also suffer from noise ${ }^{40,41}$, the concept of scAR might help design methods for the background correction. The second example might be scifiRNA-seq ${ }^{42}$, which leverages cell indexing technologies to encapsulate and sequence multiple cells in a droplet to achieve ultra-high-throughput. One can imagine intense noise in this complex setting, where scAR may be beneficial for signal denoising. 


\section{Reference}

1. Hill, A. J. et al. On the design of CRISPR-based single-cell molecular screens. Nat. Methods 15, 271-274 (2018).

2. Datlinger, P. et al. Pooled CRISPR screening with single-cell transcriptome readout. Nat. Methods 14, 297-301 (2017).

3. Lopes, R. et al. Systematic dissection of transcriptional regulatory networks by genome-scale and single-cell CRISPR screens. Sci. Adv. 7, 1-16 (2021).

4. Stoeckius, M. et al. Simultaneous epitope and transcriptome measurement in single cells. Nat. Methods 14, 865-868 (2017).

5. Mimitou, E. P. et al. Multiplexed detection of proteins, transcriptomes, clonotypes and CRISPR perturbations in single cells. Nat. Methods 16, 409-412 (2019).

6. Young, M. D. D. \& Behjati, S. SoupX removes ambient RNA contamination from droplet-based single-cell RNA sequencing data. Gigascience 9, 303727 (2020).

7. Yang, S. et al. Decontamination of ambient RNA in single-cell RNA-seq with DecontX. Genome Biol. 21, 57 (2020).

8. Smith, T., Heger, A. \& Sudbery, I. UMI-tools: Modeling sequencing errors in Unique Molecular Identifiers to improve quantification accuracy. Genome Res. 27, 491-499 (2017).

9. Griffiths, J. A., Richard, A. C., Bach, K., Lun, A. T. L. \& Marioni, J. C. Detection and removal of barcode swapping in single-cell RNA-seq data. Nat. Commun. 9, 1-6 (2018).

10. Eraslan, G., Simon, L. M., Mircea, M., Mueller, N. S. \& Theis, F. J. Single-cell RNA-seq denoising using a deep count autoencoder. Nat. Commun. 10, 1-14 (2019).

11. Lopez, R., Regier, J., Cole, M. B., Jordan, M. I. \& Yosef, N. Deep generative modeling for single-cell transcriptomics. Nat. Methods 15, 1053-1058 (2018).

12. Gayoso, A. et al. Joint probabilistic modeling of single-cell multi-omic data with totalVI. Nat. Methods 18, 272-282 (2021).

13. Mulè, M. P., Martins, A. J. \& Tsang, J. S. Normalizing and denoising protein expression data from droplet-based single cell profiling. (2021).

14. Biddy, B. A. et al. Single-cell mapping of lineage and identity in direct reprogramming. Nature 564, 219-224 (2018).

15. Guo, C. et al. CellTag Indexing: genetic barcode-based sample multiplexing for single-cell genomics. Genome Biol. 20, 90 (2019).

16. Gehring, J., Hwee Park, J., Chen, S., Thomson, M. \& Pachter, L. Highly multiplexed single-cell RNA-seq by DNA oligonucleotide tagging of cellular proteins. Nat. Biotechnol. 38, 35-38 (2020).

17. Macosko, E. Z. et al. Highly parallel genome-wide expression profiling of individual cells using nanoliter droplets. Cell 161, 1202-1214 (2015).

18. Lun, A. T. L. et al. EmptyDrops: Distinguishing cells from empty droplets in droplet-based single-cell RNA sequencing data. Genome Biol. 20, 1-9 (2019).

19. Muskovic, W. \& Powell, J. E. DropletQC: improved identification of empty droplets and damaged cells in single-cell RNA-seq data. Genome Biol. 22, 329 (2021).

20. Grønbech, C. H. et al. ScVAE: Variational auto-encoders for single-cell gene expression data. Bioinformatics 36, 4415-4422 (2020).

21. Rezende, D. J., Mohamed, S. \& Wierstra, D. Stochastic backpropagation and approximate inference in deep generative models. 31st Int. Conf. Mach. Learn. ICML 2014 4, 3057-3070 (2014).

22. Ranganath, R., Gerrish, S. \& Blei, D. M. Black box variational inference. J. Mach. Learn. Res. 33, 814-822 (2014).

23. Blei, D. M., Kucukelbir, A. \& McAuliffe, J. D. Variational Inference: A Review for Statisticians. J. Am. Stat. Assoc. 112, 859-877 (2017).

24. McDonald, E. R. et al. Project DRIVE: A Compendium of Cancer Dependencies and Synthetic Lethal Relationships Uncovered by Large-Scale, Deep RNAi Screening. Cell 170, 577-592.e10 (2017).

25. Tsherniak, A. et al. Defining a Cancer Dependency Map. Cell 170, 564-576.e16 (2017).

26. 10x genomics. Datasets 10x Genomics. https://www.10xgenomics.com/resources/datasets. (2019).

27. Genga, R. M. J. et al. Single-Cell RNA-Sequencing-Based CRISPRi Screening Resolves Molecular Drivers of Early Human Endoderm Development. Cell Rep. 27, 708-718.e10 (2019).

28. Dixit, A. et al. Perturb-Seq: Dissecting Molecular Circuits with Scalable Single-Cell RNA 
Profiling of Pooled Genetic Screens. Cell 167, 1853-1866.e17 (2016).

29. Jaitin, D. A. et al. Dissecting Immune Circuits by Linking CRISPR-Pooled Screens with SingleCell RNA-Seq. Cell 167, 1883-1896.e15 (2016).

30. Tian, R. et al. CRISPR Interference-Based Platform for Multimodal Genetic Screens in Human iPSC-Derived Neurons. Neuron 104, 239-255.e12 (2019).

31. Meyers, R. M. et al. Computational correction of copy number effect improves specificity of CRISPR-Cas9 essentiality screens in cancer cells. Nat. Genet. 49, 1779-1784 (2017).

32. Dempster, J. M. et al. Extracting Biological Insights from the Project Achilles Genome-Scale CRISPR Screens in Cancer Cell Lines. bioRxiv 720243 (2019) doi:10.1101/720243.

33. Fleming, S. J., Marioni, J. C. \& Babadi, M. CellBender remove-background: a deep generative model for unsupervised removal of background noise from scRNA-seq datasets. bioRxiv 791699 (2019) doi:10.1101/791699.

34. Tang, W. et al. BayNorm: Bayesian gene expression recovery, imputation and normalization for single-cell RNA-sequencing data. Bioinformatics 36, 1174-1181 (2020).

35. Hafemeister, C. \& Satija, R. Normalization and variance stabilization of single-cell RNA-seq data using regularized negative binomial regression. Genome Biol. 20, 1-15 (2019).

36. Lause, J., Berens, P. \& Kobak, D. Analytic Pearson residuals for normalization of single-cell RNA-seq UMI data. Genome Biol. 22, 1-20 (2021).

37. Gayoso, A. et al. A Python library for probabilistic analysis of single-cell omics data. Nat. Biotechnol. (2022) doi:10.1038/s41587-021-01206-w.

38. Duan, B. et al. Model-based understanding of single-cell CRISPR screening. Nat. Commun. 10, 2233 (2019).

39. Yang, L. et al. ScMAGeCK links genotypes with multiple phenotypes in single-cell CRISPR screens. Genome Biol. 21, 1-14 (2020).

40. Lareau, C. A. et al. Droplet-based combinatorial indexing for massive-scale single-cell chromatin accessibility. Nat. Biotechnol. 37, 916-924 (2019).

41. Chen, H. et al. Assessment of computational methods for the analysis of single-cell ATAC-seq data. Genome Biol. 20, 1-25 (2019).

42. Datlinger, P. et al. Ultra-high-throughput single-cell RNA sequencing and perturbation screening with combinatorial fluidic indexing. Nat. Methods 18, 635-642 (2021).

43. Kingma, D. P. \& Welling, M. Auto-encoding variational bayes. 2nd Int. Conf. Learn. Represent. ICLR 2014 - Conf. Track Proc. 1-14 (2014).

44. Kullback, S. \& Leibler, R. A. On Information and Sufficiency. Ann. Math. Stat. 22, 79-86 (1951).

45. Blundell, C., Cornebise, J., Kavukcuoglu, K. \& Wierstra, D. Weight uncertainty in neural networks. 32nd Int. Conf. Mach. Learn. ICML 2015 2, 1613-1622 (2015).

46. Kingma, D. P. \& Ba, J. L. Adam: A method for stochastic optimization. in 3rd International Conference on Learning Representations, ICLR 2015 - Conference Track Proceedings (2015).

47. Wolf, F. A., Angerer, P. \& Theis, F. J. SCANPY: Large-scale single-cell gene expression data analysis. Genome Biol. 19, (2018). 


\section{Methods}

\section{The scAR model.}

SCAR uses a hypothesis-driven probabilistic deep learning approach to infer the biological and technical variation in droplet-based single-cell omics experiments. The observed raw counts are modelled as a combination of biological signals and technical artefacts.

\section{Modelling count data using Binomial regression.}

We take a generative approach to modelling the observed count matrix $X \in \mathbb{N}_{0}{ }^{M \times G}$, which denotes $M$ cells and $G$ features (i.e., genes, antibodies, sgRNAs or identity barcodes). A graphical model representation of this generative model is summarised in supplementary Fig. 11. For a given cell $m, x_{m}$ represents a G-dimensional vector of observed expression data. We assume that $x_{m}$ is drawn from a Multinomial model:

$$
x_{m} \sim \operatorname{Multinomial}\left(d_{m}, \operatorname{prob}=\theta_{m}\right)
$$

where $d_{m}$ is the library size of cell $m$ and $\theta_{m}$ is the feature frequency vector of size G. $d_{m}$ is expected to be large enough to use a Poisson approximation for the multinomial. Therefore, for feature $g$ in cell $m$, the observed count $x_{m g}$ is drawn from a Binomial model:

$$
x_{m g} \sim \operatorname{Binomial}\left(d_{m}, \operatorname{prob}=\theta_{m g}\right)
$$

where $\theta_{m g}$ represents the probability of observing feature $g$ in cell $m$. It is determined by two factors, the native expression $n_{m g}$ and the ambient signals $a_{m g}$, which can be modelled as,

$$
\begin{aligned}
& \theta_{m g}=n_{m g}+a_{m g} \\
& n_{m g}=\left(1-\varepsilon_{m}\right) \times \beta_{m g} \\
& a_{m g}=\varepsilon_{m} \times \alpha_{m g}
\end{aligned}
$$

where $\varepsilon_{m} \in[0,1]$ is a hidden variable, representing the fraction of total ambient counts. $\beta_{m g}$ is another hidden variable, representing the feature frequency of the true native expression of feature $g$ in cell $m . \alpha_{m g}$ represents the ambient frequency and according to ambient signal hypothesis, it is independent of cells, so we get,

$$
\alpha_{m g}=\alpha_{g}
$$

Notably, the cell-free droplets can be expressed as in equation (2), with the native component being zero and noise ratio being 1 , so, 


$$
x_{e g}^{\prime} \sim \operatorname{Binomial}\left(d_{e}^{\prime}, \text { prob }=\alpha_{g}\right)
$$

Where, $x_{e g}^{\prime}$ and $d_{e}^{\prime}$ represent counts for feature $g$ and library size in cell-free droplet $e$, respectively. According to the law of large numbers, we can approximate $\alpha_{g}$ by averaging feature counts in cell-free droplets,

$$
\alpha_{g}=\frac{\sum_{E} x_{e g}^{\prime}}{\sum_{E} \sum_{G} x_{e g}^{\prime}}
$$

Put all together, we have,

$$
x_{m g} \sim \operatorname{Binomial}\left(d_{m}, \text { prob }=\left(1-\varepsilon_{m}\right) \times \beta_{m g}+\varepsilon_{m} \times \frac{\sum_{E} x_{e g}^{\prime}}{\sum_{E} \sum_{G} x_{e g}^{\prime}}\right)
$$

where only $\varepsilon_{m}$ and $\beta_{m g}$ are unknown parameters that need to be estimated. According to Bayes' theorem, we get the posterior probability,

$$
p\left(\varepsilon_{m}, \beta_{m g} \mid x_{m g}\right)=\frac{p\left(x_{m g} \mid \varepsilon_{m}, \beta_{m g}\right) \times p\left(\varepsilon_{m}, \beta_{m g}\right)}{p\left(x_{m g}\right)}
$$

Since the prior probability $p\left(\varepsilon_{m}, \beta_{m g}\right)$ and likelihood $p\left(x_{m g} \mid \varepsilon_{m}, \beta_{m g}\right)$ are both generally intractable, we implement a variational inference ${ }^{23,43}$ approach to estimate $\varepsilon_{m}$ and $\beta_{m g}$, as described in the following section. To ensure flexibility, we also provide implementations without the Poisson approximation to allow users to choose and test.

\section{Variational inference for scAR}

We apply variational autoencoder strategy to estimate the hidden variable $\varepsilon_{m}$ and $\beta_{m g}$ mentioned above. The architecture of the VAE is demonstrated in supplementary Fig. 12. VAEs introduce an additional latent variable $z$ in the bottleneck layer so the marginal log-likelihood of observation $x_{m}$ can then be written as,

$$
\log p_{\varphi}\left(x_{m}\right)=-\log p_{\varphi}\left(z, \varepsilon_{m}, \beta_{m} \mid x_{m}\right)+\log p_{\varphi}\left(z, \varepsilon_{m}, \beta_{m}, x_{m}\right)
$$

where $\varphi$ represents the parameter space, i.e., model weights. $\varepsilon_{m}$ and $\beta_{m}$ are calculated by a deterministic neural network (i.e., the decoder),

$$
\varepsilon_{m}, \beta_{m}=f_{\eta}(z)
$$

where, $f$ represents the decoder and $\eta \subset \varphi$ represents the trainable weights of $f$. This means,

$$
p_{\eta}\left(\varepsilon_{m}, \beta_{m} \mid z\right)=1
$$

Therefore, we can integrate out $\varepsilon_{m}$ and $\beta_{m}$ and re-write the equation (13) as

$$
\log p_{\varphi}\left(x_{m}\right)=-\log p_{\varphi}\left(z \mid x_{m}\right)+\log p_{\varphi}\left(z, x_{m}\right)
$$


We construct variational posterior $q(\varphi \mid \omega)$ to approximate the posterior $p\left(\varphi \mid x_{m}\right)$.

Therefore, we have,

$$
\log p_{\varphi}\left(x_{m}\right)=\mathbb{E}_{z \sim q_{\omega}}\left[\log \frac{q_{\omega}\left(z \mid x_{m}\right)}{p_{\varphi}\left(z \mid x_{m}\right)}\right]+\mathbb{E}_{z \sim q_{\omega}}\left[\log p_{\varphi}\left(z, x_{m}\right)-\log q_{\omega}\left(z \mid x_{m}\right)\right]
$$

where the first term on the right side is the Kullback-Leibler divergence ${ }^{44,45}$ between distributions $q$ and $p$, reflecting the difference between parameter distributions. It is non-negative, so we can get the evidence lower bound (ELBO) as follows,

$$
\log p_{\varphi}\left(x_{m}\right) \geq \mathbb{E}_{z \sim q_{\omega}}\left[\log p_{\varphi}\left(z, x_{m}\right)-\log q_{\omega}\left(z \mid x_{m}\right)\right]=: \mathcal{L}\left(\varphi, \omega ; x_{m}\right)
$$

Increasing the ELBO will approximate the distribution $q$ to $p$ thereby ensuring the learnt variables are as close as the expectation. Therefore, the ELBO is generally used as the objective function to fit the VAE. We can further transformation equation (18) into,

$$
\mathcal{L}\left(\varphi, \omega ; x_{m}\right)=-D_{K L}\left(q_{\omega}\left(z \mid x_{m}\right) \| p_{\varphi}(z)\right)+\mathbb{E}_{z \sim q_{\omega}}\left[\log p_{\varphi}\left(x_{m} \mid z\right)\right]
$$

The negative of ELBO is used as loss function to simultaneously optimize model weights and hidden variables in scAR. In case of M cells, the loss function is then written,

$$
\text { Loss }=D_{K L}\left(q_{\omega}(z \mid x) \| p_{\varphi}(z)\right)-\mathbb{E}_{z \sim q_{\omega}}\left[\sum_{i}^{M} \log p_{\varphi}\left(x_{i} \mid z\right)\right]
$$

Minimizing the loss function requires a tradeoff between the $\mathrm{KL}$ divergence and expected negative log-likelihood term. On the one hand, the KL divergence between $q_{\omega}(z \mid x)$ and $p_{\varphi}(z)$ should be kept small, preventing the variational posterior from being too different to the prior. On the other, the variational posterior parameters should maximize the log likelihood $\log p_{\varphi}(x \mid z)$, ensuring a small reconstruction error of scAR. We use the reparameterization trick to calculate the gradients with respect to $\varphi$ and $\omega$ for $\mathrm{KL}$ term ${ }^{45}$. According to equations (14) and (15), we have,

$$
p_{\varphi}\left(x_{m} \mid z\right)=p_{\varphi}\left(x_{m} \mid \varepsilon_{m}, \beta_{m}\right)
$$

Since we assume $x_{m}$ is drawn from Binomial distribution with latent parameters $\varepsilon_{m}, \beta_{m}$ (see equation (9)), $p_{\varphi}\left(x_{m} \mid \varepsilon_{m}, \beta_{m}\right)$ also has a closed-form expression, thus the gradient descents of negative log-likelihood term in equation (20) are easy to calculate. Together, we use the gradients of the loss function to update the parameters $\varphi$ and $\omega$ to determine the hidden variables noise ratio $\varepsilon$ and expected native frequencies $\beta$.

Bayesian inference and assignment of identity barcode 
We infer the expected native signals $\bar{n}_{m}$ and ambient signals $\bar{a}_{m}$ in cell $m$ using the following equations,

$$
\begin{aligned}
& \bar{n}_{m}=\left(1-\varepsilon_{m}\right) \times d_{m} \times \beta_{m} \\
& \bar{a}_{m}=\varepsilon_{m} \times d_{m} \times \alpha
\end{aligned}
$$

where $\bar{n}_{m}$ is used as the denoised counts.

Furthermore for the assignment of sgRNAs in CROP-seq, we also use and provide Bayesian factor as a metric to compare two hypotheses: the observed counts consist of both native and ambient sgRNAs $\left(H_{1}\right)$ vs the observed counts contain only ambient sgRNAs $\left(H_{2}\right)$. For a given sgRNA $g$ in cell $m$, this can be mathematically expressed as follows,

$$
\begin{gathered}
H_{1}^{m g}:=x_{m g} \sim \operatorname{Binomial}\left(d_{m}, \text { prob }=\bar{a}_{m g}+\bar{n}_{m g}\right) \text { versus } H_{2}^{m g}:= \\
x_{m g} \sim \operatorname{Binomial}\left(d_{m}, \text { prob }=\bar{a}_{m g}\right)
\end{gathered}
$$

The Bayesian factor is then given by,

$$
K_{m g}=\frac{\operatorname{Pr}\left(x_{m g} \mid H_{1}^{m g}\right)}{\operatorname{Pr}\left(x_{m g} \mid H_{2}^{m g}\right)}
$$

The numerator and denominator represent the probability that $x_{m g}$ is produced under assumption of $H_{1}^{m g}$ and $H_{2}^{m g}$, and we approximate them using the cumulative distribution function (stats.binom.cdf) and probability mass function (stats.binom.pmf), respectively. High $K_{m g}(>=3)$ favours the first hypothesis, meaning the sgRNA $g$ contains native signal. In the case of multiple high $K$, we assign the sgRNA of highest $K$ to the cell.

\section{Model optimisation for ScAR}

To identify a universal set of hyperparameters as the default setting for scAR, we perform grid search on two types of synthetic datasets (see supplementary note I), which simulate CROP-seq data type and CITE-seq/scRNAseq data type respectively. To limit the number of parameters, we fix several less important parameters. For example, the training epochs are fixed at 800. Additionally, we use the Adam optimizer $^{46}$ with exponential decay to schedule the learning rate but the decay rate is fixed at 0.97 every 5 epochs. The hyperparameters which are optimised include the number of nodes of neural networks, the dimension of latent space, the dropout probability of neurons, the initial learning rate, and the $\mathrm{KL}$ divergence weight. As a result, we tested 6912 combinations of parameters for each dataset (supplementary 
Fig. 13) and identified the most appropriate set listed as follows: units of 1st layer: 150; units of 2nd layer: 100, dimension of latent space: 15; initial learning rate: 0.001 , dropout probability: 0; KLD weight: 1e-5. All experiments were performed using these optimised parameters unless otherwise specified.

\section{CROP-seq experiment.}

The CROP-seq library was cloned into a modified pLKO-TET-ON plasmid in a pooled format by Golden Gate. The cloning reaction product was used to transform Endura electrocompetent cells, which were expanded in LB medium overnight $($ OD600 $=0.8)$ and plasmid DNA was harvested using Genopure plasmid maxi kit (Roche). We produced lentiviral particles and transduced MCF7-dCas9-KRAB cells $(\mathrm{MOI}=0.3)$ with the CROP-seq library. The cells were selected with $2 \mu \mathrm{g} / \mathrm{ml}$ puromycin (Invitrogen) and they harvested at defined time points by FACS (mCherrypositive cells). The single-cell suspensions were fixed in $90 \%$ methanol in DPBS (v/v) and stored at $-80{ }^{\circ} \mathrm{C}$ prior to rehydration and further processing. The rehydration buffer was supplemented with $1 \%$ Bovine serum albumin and $0.5 \mathrm{U} / \mathrm{ul}$ RNase inhibitor (Sigma, P/N 3335399001). All samples were processed using Chromium Next GEM single-cell 3' reagents kit (10x Genomics) according to the manufacturer's protocol and the libraries were sequenced in an Illumina HiSeq 2500.

\section{Pooled CRISPR screen.}

MCF7-CRISPRi cells were transduced with independent lentiviral pools $(\mathrm{MOI}=0.3)$ of the CROP-seq library. To guarantee a correct representation of all sgRNAs in the cell population we transduced $\approx 1000$ cells per plasmid. The cells were selected using $2 \mu \mathrm{g} / \mathrm{ml}$ puromycin (Invitrogen) at 24 hours post-transduction, after which they were expanded and harvested at indicated time points. We extracted gDNA from the cells using DNeasy kit (Qiagen) and prepared libraries for next generations sequencing.

\section{Analysis of CROP-seq data.}

Single-cell sequencing data were processed using Cell Ranger (version 3.1.0, 10x Genomics) and sgRNA count matrices were generated using KITE (https://github.com/pachterlab/kite). Human genome assembly (Ensembl GRCh38 release-98) was used as the reference to map mRNA reads. The Scanpy package ${ }^{47}$ 
was used to perform quality control, cell filtering, gene filtering and differential expression analysis. We used two normalisation approaches to examine the knockdown effect. The first one as shown in Fig. $2 g-i$ is a library size normalisation. Sequencing depth per cell was normalised to $1.0 \mathrm{xe}^{5}$ counts and t-test was performed on the normalised counts across cell groups using the scipy.stats.ttest_ind function. The second one (supplementary Fig. 3a) is a Z-normalisation as reported in our previous publication ${ }^{3}$. For each gene, we subtracted the mean value of CTL group then divided by the standard deviation of the CTL group.

\section{Analysis of CITE-seq data.}

The cellranger outputs of PBMCs $5 \mathrm{k}^{26}$ dataset were downloaded from 10x genomics. Cells with extreme counts (<1500 counts or >15000 counts) were discarded. Stressed cells with high presence of mitochondrial genes $(>=0.2)$ were also discarded. The cell clustering was performed using Scanpy and annotated based on expression of a panel of marker genes.

Correlation of RNA-protein pairs. Spearman's correlation was performed between RNA and protein counts using scipy.stats.spearmanr function. Control antibodies were removed for this correlation analysis. CD45RA and CD45RO, which are encoded by an identical gene PTPRC were also removed due to the difficulty of identifying isoform transcripts. In addition, several markers (CD15, CD34, CD80, CD137, CD274, CD278, PD-1) were removed due to extremely low counts of either protein or corresponding RNAs.

\section{Species-mixing experiment.}

The cellranger outputs of species-mixing dataset ${ }^{26}$ were downloaded from 10x genomics. Scanpy was used to perform quality control, gene filtering, cell filtering and species identification. In brief, we first took the 'filtered_feature_bc_matrix' from cellranger output and further filtered out genes with extreme counts $(<200$ or $>6000$ in total) and cells with low gene counts $(<200)$. We then performed library size normalization, log transformation, clustering and UMAP. By checking the differently expressed genes, we identified 7590 HEK293T cells, 8005 NIH3T3 cells as well as 697 multiplets mixed with both HEK293T and NIH3T3.

Examination of droplets. To identify the best representation of ambient signals, we examined subpopulations of droplets in the unfiltered matrix - namely, 
'raw_feature_bc_matrix'. All droplets were ranked by their total UMI counts and split into four subgroups through kneeplot: 1) droplets in 'filtered_feature_bc_matrix' were marked as cells, 2) droplets with high counts (>40) were marked as 'droplet I', 3) droplets with intermediate counts (>12 and <=40) were marked as 'droplet II', 4) droplets with low counts (<=12 and $>0$ ) were marked as 'cell-free droplets'. We took the total gene frequencies in each subpopulation as the ambient frequencies and run scAR to compare the estimated noise ratio.

\section{Benchmarking efficiency.}

For benchmarking the model size, we generated synthetic CITEseq data (supplementary note I), run the models for 4 epochs, and counted the trainable parameters. For benchmarking the runtime, we downloaded a 1.3 million single cell dataset from 10x genomics, selected top 5000 highly variable genes, randomly sampled subpopulations of various sample size, and run the models with identical settings. We turned off the early stopping and fixed at 100 epochs with batch size of 128. We took 0.99 of the total samples as training set. Default values were used for other parameters. We run the benchmarking experiments using one 28-core Intel E5-2690 CPU (500 GB RAM), and one NVIDIA Tesla P100 GPU (16 GB RAM). scAR, totalVI, and scVI all were run on the GPU, and DCA were run using all 28-core CPU.

\section{Data availability.}

The CROP-seq data discussed in this manuscript have been deposited to the Sequence Read Archive and will be accessible through BioProject accession number: PRJNA794328. All other datasets are public. The CITE-seq datasets (PBMCs5k) and HEK293T and NIH3T3 pooled scRNAseq (20k_hgmm dataset) were downloaded from 10x genomics datasets. Other datasets were downloaded from Sequence Read Archive.

\section{Code availability.}

The scAR package is available at GitHub (https://github.com/CaibinSh/scAR). The full codes to reproduce the results in this manuscript will be available at GitHub upon publication (https://github.com/CaibinSh/scAR-reproducibility). 


\section{Acknowledgements.}

We thank Joel Wagner, Joshua Korn, Viveksagar Krishnamurthy Radhakrishna, and Jeanne Whalen for inspiring discussions and testing scAR, Mathias Eder and Esther Uijttewaal for additional technical support.

\section{Author contributions.}

C.S. and A.d.W. conceived and designed the study. C.S., G.L. and A.d.W. designed the statistical model and performed analysis. R.L. and G.G.G. designed CROP-seq and bulk sequencing experiments and R.L. conducted the experiments. A.W. and R.C. performed scRNAseq and bulk sequencing and S.S. performed preprocessing of CROP-seq data. S.D., A.K., E.D., G.G.G, G.R. and A.d.W. supervised the study. C.S. wrote the original draft. C.S., G.L., R.L., S.D., E.D., G.G.G, G.R. and A.d.W. reviewed and edited the draft. 